\title{
Clinical Significance of Circulating Tumor Cells in Pancreatic Cancer
}

\author{
Guangfu Li ${ }^{1,2^{*}}$, Yue Huang ${ }^{1,2}$, Yariswamy Manjunath ${ }^{1,2}$, Eric T Kimchi ${ }^{1,2}$, Jussuf T Kaifi ${ }^{1,2}$ and Kevin F Staveley-O'Carroll ${ }^{1,2,3^{*}}$
}

${ }^{1}$ Department of Surgery, University of Missouri, Columbia, Missouri, United States of America

${ }^{2}$ Ellis Fischel Cancer Center, University of Missouri, Columbia, Missouri, United States of America

${ }^{3}$ Department of Molecular Microbiology and Immunology, University of Missouri, Columbia, Missouri, United States of America

"Corresponding authors: Guangfu Li, Ph.D, DVM, Assistant Professor, Department of Surgery, Ellis Fischel Cancer Center, The University of Missouri, One Hospital Drive, Columbia, Missouri 65212, United States of America, Tel: 573-882-8454; Fax: 573-884-6054; E-mail: liguan@health.missouri.edu

Kevin F Staveley-O'Carroll, M.D, Ph.D, Professor and Chair of Surgery, Director of Ellis Fischel Cancer Center, The University of Missouri, One Hospital Drive, Columbia, Missouri 65212, United States of America, Tel: 573-882-8454; Fax: 573-884-6054; E-mail: ocarrollk@missouri.edu

Received date: October 29, 2016; Accepted date: November 17, 2016; Published date: November 24, 2016

Copyright: (C) 2016 Li G, et al. This is an open-access article distributed under the terms of the Creative Commons Attribution License, which permits unrestricted use, distribution and reproduction in any medium, provided the original author and source are credited.

\begin{abstract}
Pancreatic cancer (PC) is a highly aggressive malignancy with limited therapeutic options. Early and accurate detection is critically important for successful cancer treatment. Currently, the most widely used and best validated marker for pancreatic cancer is carbohydrate antigen 19-9 (CA 19-9) and carcinoembryonic antigen (CEA). However, inadequate sensitivity and specificity limit the use of CA 19-9 and CEA in the early screening and diagnosis of pancreatic cancer. During past ten years, circulating tumor cells (CTCs) as a new liquid biomarker have received enormous attention in cancer diagnosis and management. Although CTCs are extremely rare and challenging to be isolated, their clinical significance has extensively been investigated in numerous clinical trials. In this review, we summarize the CTC application in translational pancreatic cancer research and its outlook in the future, specifically evaluating its clinical potential in pancreatic cancer diagnosis, prognosis, and treatments. Development of sensitive, specific, and reliable methods is expected towards a major impact on our ability in cancer management.
\end{abstract}

Keywords: Pancreatic cancer (PC); Circulating tumor cells (CTCs); Biomarker; Clinical trials

\section{Introduction}

Pancreatic cancer (PC) is a highly aggressive malignancy with an average 5-year survival rate of $<6 \%$ worldwide [1,2]. The most common type is pancreatic ductal adenocarcinoma (PDAC), representing around $90 \%$ of PCs [3]. According to the latest cancer statistics report from the American Cancer Society which is published in CA: A Cancer Journal for Clinicians [3], about 53,000 new cases of PC will be diagnosed in the US in 2016, causing around 42,000 deaths. PC is expected to surpass colorectal cancer to become the third leading cause of cancer-related death in the United States if current trend continues. Frequent and early dissemination of the disease, drug resistance, as well as late detection result in poor prognosis of PC patients [4]. The lack of progress in primary prevention, early diagnosis, metastases detection, and treatment underscores more efforts is needed in PC research [5,6].

Current biomarkers are insufficient in PC clinical management. Serum or tissue tumor markers have been proposed for use in clinical practice to predict prognosis, monitor response to treatment, and help detect recurrence or metastases. Among these biomarkers, carbohydrate antigen 19-9 (CA 19-9) [7] and carcinoembryonic antigen (CEA) [8] are the only blood-based biomarkers which are most widely investigated in clinical application. However, elevation of serum CA 19-9 and CEA is mostly found in patients with advanced and incurable PC. In addition, elevated levels of these tumor markers can be detected in individuals without PC. Low sensitivity and specificity limit their application for clinical screening. In clinical practice, these tests are most useful to predict treatment response and cancer progression. Several other markers have been studied. Some studies have shown that CA19-9 and CA242 have similar sensitivity in the diagnosis of PC, which is higher than CEA [9]. However, no single marker has been shown to be better than CA19-9 [10].

Circulating tumor cells (CTCs) is emerging as one of the most promising liquid biomarkers with broad clinical application in cancer management. Thomas Ashworth, an Australian physician, firstly found CTCs in the blood of a patient with metastatic cancer in 1869. These cells are similar to the primary tumor cells identified in the blood vessels of autopsied cancer patients [11]. Sampling of peripheral blood CTCs provides a relatively non-invasive and real-time means to easily obtain reproducible liquid biopsy. These advantages highlight CTC's important value in clinical application [12]. Unceasing studies demonstrate that CTC is extremely rare as one milliliter of human blood carries approximately one billion red blood cells, seven million white blood cells, and 300 million platelets, but only about 1-10 CTCs [13]. In addition, no unique or CTC-specific markers can be used to isolate these cells. Therefore, detection and characterization of CTCs are still a challenging task. During the past more than ten years, technique advancement in the field of CTC has been taking place. Studies on CTCs have received enormous attention [14]. So far, large patient series of breast, prostate, lung, colon cancer, and PC have been testing for CTCs $[15,16]$. Searching for the term "circulating tumor cell" at https://clinicaltrials.gov/ yields 823 clinical studies. Among them, 23 are of pancreatic cancer. Here, we categorize these ongoing or completed CTC clinic trials in PC, analyzing the reported results, and evaluate their potential in clinical utility towards advancing their future translational cancer research. 


\section{Rapid expansion of CTC clinical trials in PCs}

CTCs are the cells that shed into the vasculature from a primary tumor or metastases and circulate in the bloodstream. As a new realtime and liquid biomarker, CTCs provide an ideal means to increase our knowledge in tumor formation, progression, and metastasis. In addition, detection of CTCs can improve the early tumor detection and diagnosis, guide therapeutic choices. Therefore, the value of CTC as a new effective and reliable tool has received enormous attention and becomes one of the most active areas of translational cancer research.

In 2006, Dr. Sergeant and his colleagues registered the first CTC trial in PC in clinicaltrials.gov (NCT00495924). The aim of this study is to develop a CTC gene signature for assessing its prognostic relevance with surgical operation in PDAC patients [17]. Using FACS-based method, CTCs was enriched from the blood of 10 patients who underwent surgery for PDAC. Total RNA was isolated from four different samples including CTCs, hematological cells $(G)$, original tumors $(\mathrm{T})$, and non-tumoral pancreatic control tissues $(\mathrm{P})$. Whole genome microarray detected 8,152 genes, their functional analysis was conducted with 'Ingenuity Pathway Analysis' software and AmiGO. Finally, a CTC gene signature was developed and validated with the nCounter system on expression data of 78 primary PDACs. The results demonstrate that the defined cell motility gene signature in the primary tumor can predict survival of patients undergoing surgical resection for PC. Thereafter, more and more CTC clinical trials were requested and conducted. From 2001 to present, in total 23 registered CTC studies in PC were found in clinical trial (Table 1). Among them, around $80 \%$ trials were performed after 2010 (Figure 1). This rapid increase in CTC clinical trials for PC is similar to translational research in other types of cancers. The rapidly rising trend highlights the value, importance, and clinical significance of CTCs.

\begin{tabular}{|c|c|c|c|c|}
\hline Registered No. & Clinical Utility & Start year & Sponsor & Status \\
\hline NCT00495924 & Early tumor recurrence & 2006 & Katholieke Universiteit Leuven & Completed \\
\hline NCT00898781 & Tumor progression & 2007 & Mayo Clinic & Terminated \\
\hline NCT00524069 & Neoadjuvant therapy efficacy & 2007 & Roswell Park Cancer Institute & Withdrawn \\
\hline NCT00967603 & Chemotherapy efficacy & 2008 & IRCCS San Raffaele & Completed \\
\hline NCT01919151 & Tumor initiation and progression & 2008 & Oslo University Hospital & Unknown \\
\hline NCT01116791 & Chemotherapy efficacy & 2010 & Baki Topal, University Hospital, Gasthuisberg & Terminated \\
\hline NCT01313442 & Genetic profiling & 2011 & National Cancer Institute ( $\mathrm{NCl}$ ) & Recruiting \\
\hline NCT01474564 & Genetic profiling & 2011 & Memorial Sloan Kettering Cancer Center & Recruiting \\
\hline NCT01528774 & Tumor progression & 2012 & $\begin{array}{l}\text { Wolfram Samlowski, Comprehensive Cancer } \\
\text { Centers of Nevada }\end{array}$ & Active, not recruiting \\
\hline NCT01783054 & Genetic profiling & 2012 & Medical University of South Carolina & Terminated \\
\hline NCT02406846 & $\begin{array}{l}\text { Surgical and chemotherapeutic } \\
\text { efficacies }\end{array}$ & 2013 & Fuda Cancer Hospital, Guangzhou & Completed \\
\hline NCT01915225 & Tumor diagnosis and treatment & 2013 & National Cancer Institute (NCl) & Recruiting \\
\hline NCT02305810 & Tumor progression & 2013 & European Institute of Oncology & Recruiting \\
\hline NCT02150746 & Surgical efficacy & 2014 & University of Florida & Completed \\
\hline NCT02072616 & Early diagnosis & 2014 & University Hospital, Rouen & Recruiting \\
\hline NCT02080650 & $\begin{array}{l}\text { Comparison of CTC detection } \\
\text { technology with c-MET and EpCAM }\end{array}$ & 2014 & Duke University & Recruiting \\
\hline NCT02707159 & Chemotherapy efficacy & 2014 & Helse Stavanger HF & Recruiting \\
\hline NCT02349867 & Neoadjuvant therapy efficacy & 2015 & Virginia Commonwealth University & Recruiting \\
\hline NCT02555735 & Chemotherapy efficacy & 2015 & Adera Labs, LLC & Recruiting \\
\hline NCT02451384 & Surgical efficacy & 2015 & Shanghai Zhongshan Hospital & Recruiting \\
\hline NCT02838836 & Early detection and genetic profiling & 2016 & University of Missouri-Columbia & Recruiting \\
\hline NCT02335151 & Efficacy of anesthesia & 2016 & University of Zurich & Not yet recruiting \\
\hline
\end{tabular}

Table 1: CTC clinical trials in pancreatic cancer. 


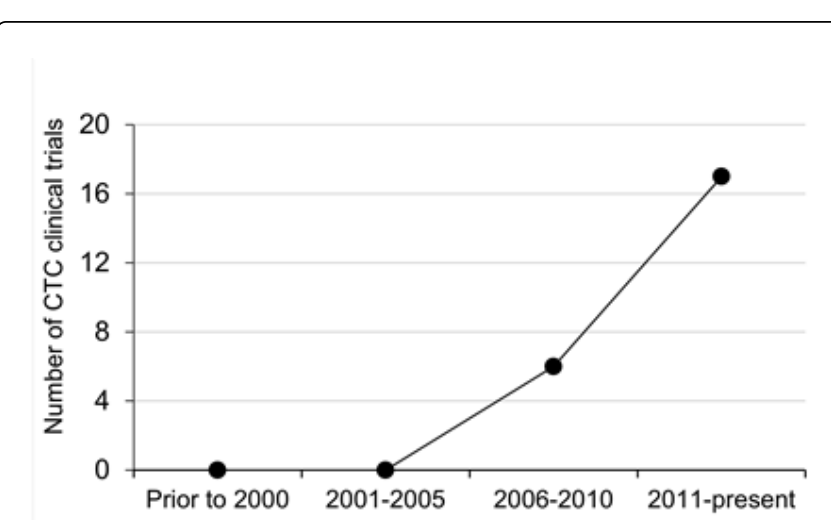

Figure 1: CTC in clinical trials of pancreatic cancer.

\section{Biological and molecular characteristics of CTCs in PC}

Our knowledge of CTCs is still very limited. Generally, CTCs are more likely to be detected in advanced tumors due to higher tumor burden. However, it was reported that CTCs could be detected in very early stage of cancer without detectable tumors [18]. Considering very short half-life [19], CTCs is assumed to capably reflect the current status of primary tumors or secondary metastatic tumors. One study group conducted the first single-cell RNA sequencing for CTC from a mouse model of PC, and identified aberrations in the WNT pathway which might potentially be involved in the metastatic cascade [20]. This finding was verified by their following studies in patients with PC [21]. Another group recently reported that 200-fold increase in the mRNA expression of plakoglobin was detected in clustered CTCs in patient with PCs compared with single CTCs [22]. These completed and ongoing biological and molecular characterization of CTCs provide new insights into pancreatic cancer and its metastasis.

\section{Categories of CTC clinical trials in terms of clinical objectives}

Currently, the clinical management of PC relies on the relatively few predictive markers including bloodborne CA 19-9 and CEA. However, these markers don't fulfill the requirement for early detection of tumor incidence and metastasis as well as a predictor of patient's response to treatments. Discovering of CTCs offer the promising tool to overcome these barriers by assessing tumor biology in real time. In terms of the objectives, a total of 23 registered CTC clinical trials fall into four major categories (Figure 2): 1) numeration of CTC as a diagnostic marker, 2) numeration of CTC as a therapeutic indicator, 3) molecular profiling of CTC, 4) new technique for CTC isolation. Among entire 23 trials, about $50 \%$ trials (11 studies) are used to predict the response to different therapies including surgical operation, chemotherapy, and adjuvant treatment. The second leading clinical application is detection of CTC for cancer diagnosis which account for $35 \%$ of total trials (8 trials). The third one is isolation of CTC for determining molecular profiles. Only one trial is to test a new CTC isolation technology with mesenchymal-marker ferrofluid (c-MET) by comparing with the FDAapproved EpCAM-based isolation. These trials demonstrate broad utility of CTC in translational cancer research.

\section{- Diagnostic marker \\ - Molecular profile \\ - Therapeutic indicator \\ - Technology development}

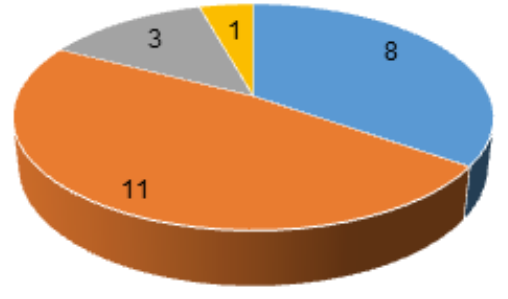

Figure 2: Category of CTC clinical trials in PC.

\section{Diagnostic values of pancreatic CTC}

Early and accurate detection of cancer is critical for successful cancer therapies. However, PC is difficult to find early. Pancreas is deep inside the body, early tumors cannot be seen or felt during routine physical exams. Patients usually have no symptoms until the tumors have already spread to other organs. Detection of CTCs within a routine blood specimen provides an opportunity to monitor cancer noninvasively, in essence a liquid biopsy [23]. Many reports have shown that pancreatic CTCs present in the peripheral blood of PDAC patients [24]. However, the reported results regarding the percentage of CTC positive patients and the number of CTCs per unit of blood vary dramatically between studies, which could be caused by diversity of the enrolled patients and the method difference used in CTC enrichment and detection [14]. Compared to other types of cancer, pancreatic cancer produces fewer CTCs in peripheral blood, evidenced by the lowest number of CTCs detected per unit of blood in PC among 12 different cancer types [24]. Catenacci et al. have reported that significantly more CTCs were detected in the portal vein blood than peripheral blood $(118.4 \pm 36.8 \mathrm{CTCs} / 7.5 \mathrm{~mL}$ vs. $0.8 \pm 0.4 \mathrm{CTCs} / 7.5$ $\mathrm{mL}, \mathrm{p}<0.1)$ [25]. Correspondingly, $100 \%$ patients were detected to be CTC positive (18 pancreatic cancer patients) with portal vein blood but only 4 out of 18 CTC positive patients were seen in peripheral blood. Similarly, $95 \%$ vs. $68 \%$ CTC detection in portal vein blood and peripheral blood was reported by another study group [26]. Also, detection of endoscopic ultrasound-guided fine needle aspiration biopsy led to diagnosis of PC in $77.8 \%$ patients while CTC detection in peripheral blood were only found in $55.5 \%$ patients [27]. These results suggest that portal vein circulation may serve as a barrier that blocks CTCs from entering systemic circulation. Thus, portal vein blood may be superior to peripheral blood in CTC detection. However, the safety of portal vein blood sampling needs to be further evaluated considering the high risk of thromboembolism in patients with PC [26]. Together, CTCs may have a significant potential in PC early screening and diagnosis.

\section{Therapeutic values of pancreatic CTCs}

An increasing body of study has shown the difference of therapeutic response among different patients receiving same anticancer treatment. But, development of reliable tools to discriminate patients likely to benefit from specific therapies is still a challenging task. Yu et al. have created a pharmacogenomic model to predict the response of a pancreatic cancer patient to chemotherapeutic agents based on the 
genetic expression signatures in pancreatic CTCs [28]. In a phase 1 study (NCT00429234), gemcitabine combined with dasatinib led to the reduced number of CTC in patients responded to the regimen compared to those without treatment response $(0.5 \pm 0.0$ vs. $3.85 \pm$ 1.57 per $7.5 \mathrm{~mL}$ blood, $\mathrm{p}=0.052$ ) [29]. The results implicate the correlation of CTC number and treatment efficacy. However, largescale further experiments with a well-designed control arm will be needed to validate this interesting result.

\section{Prognostic values of pancreatic CTCs}

The potential of pancreatic CTCs to be used as a prognostic biomarker have been extensively studied. A number of studies have reported that a higher number of CTC was correlated with a poorer clinical outcome [30,31]. In 2013, 268 CTC-positive and 355 CTCnegative patients were enrolled by 9 study groups. Meta-analysis indicated that CTC-positive patients have a worse progression-free survival (PFS) and overall survival (OS) [32]. Soeth et al. reported that detection of CTCs in either bone marrow or peripheral blood was associated with a poorer survival in patients with well- or moderatelydifferentiated pancreatic cancer [33]. In the French LAP07 clinical trial, CTCs positivity has been linked to less differentiated tumors and a poorer OS [34]. A recently published study assessed the prognostic value of epithelial-like and mesenchymal-like CTCs [35]. The results showed the epithelial-like CTCs but not the mesenchymal-like CTCs were associated with a poorer survival while the mesenchymal-like CTCs was an indicator for early tumor recurrence. However, irrelevance of CTC with survival was reported in some studies or in different setting. A phase II clinical trial in metastatic PC (NCT00474812) indicated that the number of CTCs at baseline were not associated with OS [36]. In a clinical trial (NCT00495924), surgical operation was found to increase CTC number which was not associated with a poorer clinical outcome [37]. Similarly, Gall et al. has employed a no-touch isolation technique for pancreaticoduodenectomy. Although this new technique significantly reduced CTCs postoperatively compared to the standard technique, no survival benefits were observed [38]. Bissolati et al. has found that the incidence of liver metastasis was significantly lower in patients without detectable CTCs compared to patients with CTCs ( $8 \%$ vs. $53 \%$, $\mathrm{p}=0.038$ ) [39]. However, the reduction of liver metastasis did not translate to survival benefits. In a clinical trial (NCT00495924), Sergeant et al. attempted to use gene expression signature of CTCs to predict survival for pancreatic cancer patients who underwent surgical tumor resection. The high co-expression of TGF- $\beta 1$ and a group of 9 genes function in cell motility were found to be independent markers to predict the PFS and OS [17]. Taken together, many studies have reported promising results to support the use of CTCs as a prognostic marker in PC. However, more studies are needed to define the clinical values of different subsets of CTCs in the future.

\section{Challenges and future directions}

CTC as a liquid biomarker is easily accessible and reproducible. Great effort advances its clinical application over the past decade. In addition to its inherent significance in cancer diagnosis and prognosis, molecular profiling of CTCs in a real time has provided new insights into the biology of tumor metastasis which may facilitate the development of "tailor-made" cancer treatment for an individual cancer patient. However, challenges still remain in CTC isolation and identification due to its extremely rare in the blood and technological limitation in isolation. Large-scale clinical data are still lacking. In addition, different principles for identification of CTC generate diverse results which is confusing to the cancer research. Therefore, more sensitive, specific, and reliable methods for CTC identification and isolation as well as its downstream analysis are urgently needed. In addition, it has been reported that some critical processes including epithelial mesenchymal transition (EMT) are required for primary tumor cells to differentiate into CTCs [12]. In terms of the differentiation statue, CTCs can be classified into different subpopulation including epithelial CTCs, mesenchymal CTCs, and hybrid (epithelial/mesenchymal) CTCs etc. [40,41]. Each subpopulation may have different clinical implication. It is postulated that only a specific subpopulation mediates tumor metastasis. Thus, characterization of CTC subpopulation will expand our understanding of CTCs and improve our ability in cancer management [42].

\section{Financial Support}

Dr. Kevin F. Staveley-O'Carroll would like to thank the National Cancer Institute/National Institutes of Health Grant number 1 R01 CA164335-01A1, USA for providing financial support.

\section{Conflict of Interest}

The authors have declared that no conflict of interest exists.

\section{References}

1. Miller KD, Siegel RL, Lin CC, Mariotto AB, Kramer JL, et al. (2016) Cancer treatment and survivorship statistics, 2016. Cancer J Clin 66: 271-289.

2. Ferlay J, Steliarova-Foucher E, Lortet-Tieulent J, Rosso S, Coebergh JW, et al. (2013) Cancer incidence and mortality patterns in Europe: estimates for 40 countries in 2012. Eur J Cancer 49: 1374-1403.

3. Siegel RL, Miller KD, Jemal A (2016) Cancer statistics, 2016. Cancer J Clin 66: 7-30.

4. Spadi R, Brusa F, Ponzetti A, Chiappino I, Birocco N, et al. (2016) Current therapeutic strategies for advanced pancreatic cancer: A review for clinicians. World J Clin Oncol 7: 27-43.

5. Hussain SP (2016) Pancreatic cancer: current progress and future challenges. Int J Biol Sci 12: 270-272.

6. Rath N, Olson MF (2016) Regulation of pancreatic cancer aggressiveness by stromal stiffening. Nat Med 22: 462-463.

7. Balzano G, Di Carlo V (2008) Is CA 19-9 useful in the management of pancreatic cancer? Lancet Oncol 9: 89-91.

8. Zamcheck N (1976) The present status of carcinoembryonic antigen (CEA) in diagnosis, detection of recurrence, prognosis and evaluation of therapy of colonic and pancreatic cancer. Clin Gastroenterol 5: 625-638.

9. Zhang Y, Yang J, Li H, Wu Y, Zhang H, et al. (2015) Tumor markers CA19-9, CA242 and CEA in the diagnosis of pancreatic cancer: a metaanalysis. Int J Clin Exp Med 8: 11683-11691.

10. Tanday S (2014) Biomarkers in blood could help to detect pancreatic cancer. Lancet Oncol 15: e108.

11. Ashworth TR (1869) A case of cancer in which cells similar to those in the tumours were seen in the blood after death. Australian Medical Journal 14: 146-147.

12. Plaks V, Koopman CD, Werb Z (2013) Cancer: Circulating tumor cells. Science 341: 1186-1188.

13. Mockelmann N, Laban S, Pantel K, Knecht R (2014) Circulating tumor cells in head and neck cancer: clinical impact in diagnosis and follow-up. Eur Arch Otorhinolaryngol 271: 15-21.

14. Alix-Panabieres C, Pantel K (2014) Challenges in circulating tumor cell research. Nat Rev Cancer 14: 623-631. 
15. Liberko M, Kolostova K, Bobek V (2013) Essentials of circulating tumor cells for clinical research and practice. Crit Rev Oncol Hematol 88: 338-356.

16. Kaur S, Baine MJ, Jain M, Sasson AR, Batra SK (2012) Early diagnosis of pancreatic cancer: challenges and new developments. Biomarkers Med 6: 597-612.

17. Sergeant G, van Eijsden R, Roskams T, Van Duppen V, Topal B (2012) Pancreatic cancer circulating tumour cells express a cell motility gene signature that predicts survival after surgery. BMC cancer 12: 527.

18. Ren C, Chen H, Han C, Jin G, Wang D, et al. (2013) Detection and molecular analysis of circulating tumor cells for early diagnosis of pancreatic cancer. Med Hypotheses 80: 833-836.

19. Pantel K, Speicher MR (2016) The biology of circulating tumor cells Oncogene 35: 1216-1224.

20. Yu M, Ting DT, Stott SL, Wittner BS, Ozsolak F, et al. (2012) RNA sequencing of pancreatic circulating tumour cells implicates WNT signalling in metastasis. Nature 487: 510-513.

21. Court CM, Ankeny JS, Hou S, Lin M, Song M, et al. (2015) Single cell mutational analysis of isolated circulating tumor cells in pancreatic cancer. Ann Surg Oncol 22: S169-S170.

22. Aceto N, Bardia A, Miyamoto DT, Donaldson MC, Wittner BS, et al (2014) Circulating tumor cell clusters are oligoclonal precursors of breast cancer metastasis. Cell 158: 1110-1122.

23. Bardia A, Haber DA (2014) Solidifying liquid biopsies: can circulating tumor cell monitoring guide treatment selection in breast cancer? J Clin Oncol 32: 3470-3471.

24. Allard WJ, Matera J, Miller MC, Repollet M, Connelly MC, et al. (2004) Tumor cells circulate in the peripheral blood of all major carcinomas but not in healthy subjects or patients with nonmalignant diseases. Clin Cancer Res 10: 6897-6904.

25. Catenacci DV, Chapman CG, Xu P, Koons A, Konda VJ, et al. (2015) Acquisition of portal venous circulating tumor cells from patients with pancreaticobiliary cancers by endoscopic ultrasound. Gastroenterology 149: 1794-1803.

26. Zhang Y, Wang F, Ning N, Chen Q, Yang Z, et al. (2015) Patterns of circulating tumor cells identified by CEP8, CK and CD45 in pancreatic cancer. Int J Cancer 136: 1228-1233.

27. Iwanicki-Caron I, Basile P, Toure E, Antonietti M, Lecleire S, et al. (2013) Usefulness of circulating tumor cell detection in pancreatic adenocarcinoma diagnosis. Am J Gastroenterol 108: 152-155.

28. Yu KH, Ricigliano M, Hidalgo M, Abou-Alfa GK, Lowery MA, et al (2014) Pharmacogenomic modeling of circulating tumor and invasive cells for prediction of chemotherapy response and resistance in pancreatic cancer. Clin Cancer Res 20: 5281-5289.

29. Hong DS, Choe JH, Naing A, Wheler JJ, Falchook GS, et al. (2013) A phase 1 study of gemcitabine combined with dasatinib in patients with advanced solid tumors. Investigational new drugs 31: 918-926.
30. Kurihara T, Itoi T, Sofuni A, Itokawa F, Tsuchiya T, et al. (2008) Detection of circulating tumor cells in patients with pancreatic cancer: a preliminary result. J Hepatobiliary Pancreat Surg 15: 189-195.

31. de Albuquerque A, Kubisch I, Breier G, Stamminger G, Fersis N, et al. (2012) Multimarker gene analysis of circulating tumor cells in pancreatic cancer patients: a feasibility study. Oncology 82: 3-10.

32. Han L, Chen W, Zhao Q (2014) Prognostic value of circulating tumor cells in patients with pancreatic cancer: a meta-analysis. Tumour Biol 35: 2473-2480.

33. Soeth E, Grigoleit U, Moellmann B, Roder C, Schniewind B, et al. (2005) Detection of tumor cell dissemination in pancreatic ductal carcinoma patients by CK 20 RT-PCR indicates poor survival. J Cancer Res Clin Oncol 131: 669-676.

34. Bidard FC, Huguet F, Louvet C, Mineur L, Bouche O, et al. (2013) Circulating tumor cells in locally advanced pancreatic adenocarcinoma: the ancillary CirCe 07 study to the LAP 07 trial. Ann Oncol 24: 2057-2061.

35. Poruk KE, Valero V, Saunders T, Blackford AL, Griffin JF, et al. (2016) Circulating tumor cell phenotype predicts recurrence and survival in pancreatic adenocarcinoma. Ann Surg 264: 1073-1081.

36. Chee CE, Krishnamurthi S, Nock CJ, Meropol NJ, Gibbons J, et al. (2013) Phase II study of dasatinib (BMS-354825) in patients with metastatic adenocarcinoma of the pancreas. Oncologist 18: 1091-1092.

37. Sergeant G, Roskams T, van Pelt J, Houtmeyers F, Aerts R, et al. (2011) Perioperative cancer cell dissemination detected with a real-time RT-PCR assay for EpCAM is not associated with worse prognosis in pancreatic ductal adenocarcinoma. BMC cancer 11: 47.

38. Gall TM, Jacob J, Frampton AE, Krell J, Kyriakides C, et al. (2014) Reduced dissemination of circulating tumor cells with no-touch isolation surgical technique in patients with pancreatic cancer. JAMA surgery 149: 482-485.

39. Bissolati M, Sandri MT, Burtulo G, Zorzino L, Balzano G, et al. (2015) Portal vein-circulating tumor cells predict liver metastases in patients with resectable pancreatic cancer. Tumour Biol 36: 991-996.

40. Jolly MK, Boareto M, Huang B, Jia D, Lu M, et al. (2015) Implications of the hybrid epithelial/mesenchymal phenotype in metastasis. Front Oncol 5: 155 .

41. Jolly MK, Tripathi SC, Jia D, Mooney SM, Celiktas M, et al. (2016) Stability of the hybrid epithelial/mesenchymal phenotype. Oncotarget 7: 27067-27084.

42. Ting DT, Wittner BS, Ligorio M, Vincent Jordan N, Shah AM, et al. (2014) Single-cell RNA sequencing identifies extracellular matrix gene expression by pancreatic circulating tumor cells. Cell reports 8: 1905-1918. 